\title{
Measurement-based Analysis of Doppler Characteristics for Ultra-Wideband Radio Channels in an Office Environment
}

\author{
Brecht Hanssens, Emmeric Tanghe, \\ Luc Martens and Wout Joseph \\ INTEC, Ghent University/iMinds \\ Ghent, Belgium \\ Brecht.Hanssens@INTEC.UGent.be
}

\author{
Claude Oestges \\ ICTEAM, Catholic University of Louvain \\ Louvain-la-Neuve, Belgium \\ Claude.Oestges@UCLouvain.be
}

\begin{abstract}
In this work, an analysis of the Doppler characteristics for Ultra-Wideband indoor communication is presented. Channel sounding measurements ranging from 3.1 to $10.6 \mathrm{GHz}$ were performed over the course of several days in an occupied office environment, with the help of a network analyzer. Based on these measurements, we analyze the behavior of both the Doppler spread and RMS Doppler spread in the Ultra-Wideband frequency band. Our measurements indicate a frequency-dependent behavior for both parameters, where consistent values could be measured with respect to time of observation.
\end{abstract}

\section{INTRODUCTION}

Indoor radio channels are commonly characterized by multipath propagation phenomena such as reflection, diffraction and scattering. As such, the channel transfer function includes contributions of a series of attenuated and delayed versions of the original transmitted signal. Over time, movement of transmitter, receiver or other objects present in the channel, will give rise to phase changes of the propagation paths. This movement will also broaden the frequency spectrum of the received signal, resulting in a frequency shift between the transmitted and received signal. These are referred to as Doppler shifts, and the width of their distribution is known as the Doppler spread. An accurate analysis of these characteristics is an important factor in determining the maximum possible signaling rate for coherent demodulation of the received envelope [1]. It is also paramount to improve detection, as well as to optimize transmission at the physical layer.

The objective of this work is to analyze Doppler characteristics of a dynamic office environment in the Ultra-Wideband (UWB) frequency range. We assume a fixed stationary location for both the transmit and receive antenna, which allows us to assign the time-variant behavior of the radio channel to the movement of the people present in our measurement scenario. Theoretical predictions in [2] have shown that the well known Jakes' Doppler spectrum no longer applies in such a scenario.

\section{Measurements}

\section{A. Measurement environment}

The indoor measurements were carried out in an occupied office environment of Ghent University, Belgium. In Fig. 1, the measured scenario is depicted, as well as the fixed positions of the transmit and receive antenna. The office space is approximately $8.48 \times 13.18 \times 2.67$ meters, occupied at mostly by 20 people, and furnished with several closets, desks, computers, and chairs. Two of the four walls are made of concrete, the other two are interior walls made of plasterboard. A concrete ceiling is located $74 \mathrm{~cm}$ above the dropped ceiling, the floor is a raised floor located $13 \mathrm{~cm}$ above a concrete floor.

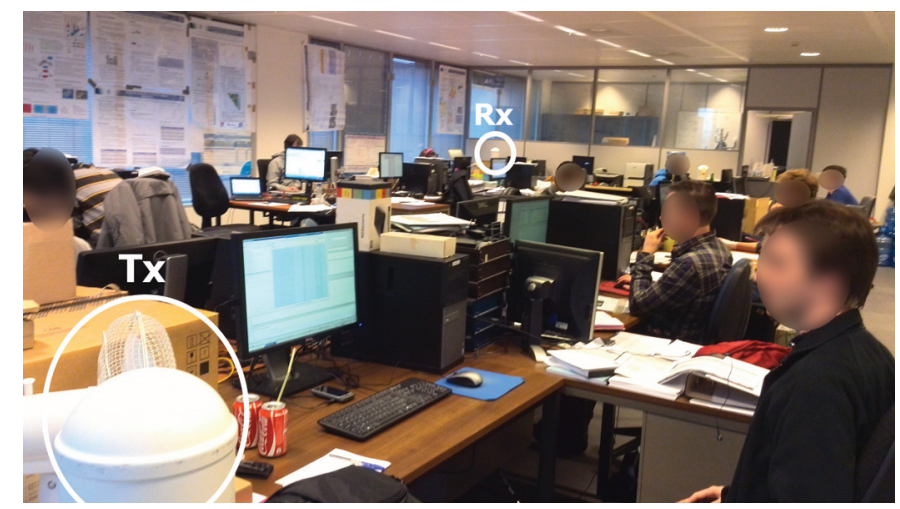

Fig. 1: Measurement scenario (Tx and Rx are indicated in white)

\section{B. Channel sounding procedure}

Channel sounding measurements were carried out in the UWB frequency range, where the complex gain $\left(S_{21}\right.$ scattering parameter) between transmit and receive antenna was measured over a range from 3.1 to $10.6 \mathrm{GHz}$ with a Vector Network Analyzer (VNA). In this frequency band, 301 uniformly spaced frequency points were sampled resulting in a spacing of $25 \mathrm{MHz}$. Each frequency was then sampled 1255 times in continuous wave mode with a sweep time of $4 \mathrm{~ms}$, resulting in a maximum measurable Doppler shift of $125 \mathrm{~Hz}$ and a time delay of $5.02 \mathrm{~s}$ between neighboring frequencies. The total measurement time was less than half an hour per such observation. During this time period, we assume that the amount of movement in the radio channel is constant over all frequencies. These observations were repeated day and night over the course of two consecutive days.

The transmitted power by the VNA was set to $10 \mathrm{~dB}$, together with an intermediate frequency of $300 \mathrm{~Hz}$ to allow for fast sweeps. Both antennas in our measurement scenario were vertically polarized XPO2V-0.3-10.0/1381 antennas by Cobham Antenna Systems, and had an omnidirectional radiation pattern in the azimuth plane. 


\section{Evaluation method}

At each of the 1255 time samples per frequency point, the VNA measures the $S_{21}$ scattering parameter (complex gain between both antennas). To further analyze these samples, we have used Welch's method [3] to calculate the Doppler power at the different possible Doppler frequencies (-125 to $125 \mathrm{~Hz}$ ). This method returns the Power Spectral Density (PSD) estimate of a discrete-time signal using Welch's averaged, modified periodogram method. This approach divides the 1255 time samples in segments with $50 \%$ overlap of a specific length, which we have set to 501 in order to obtain a Doppler resolution of $0.5 \mathrm{~Hz}$. After that, a Hannwindow is applied on each segment in the time domain before the discrete Fourier transform operation is performed. This window function was chosen for its good frequency resolution and reduced spectral leakage. The resulting periodograms are then computed and averaged, which results in a better noise reduction in the estimated power spectra in exchange for reducing the frequency resolution.

To determine the Doppler spread, one must then consider the range of Doppler frequencies over which the Doppler spectrum is non-zero. For realistic measurements, such a spectrum will never be zero due to effects such as the noise level of the VNA, measurement uncertainties, etc. As such, an alternative approach must be used. In our analysis, we have used the width of the Doppler spectrum for which the power stays above a certain threshold. This threshold was set to be the median of all powers in the Doppler- and UWB frequency domain. We have added an extra $6 \mathrm{~dB}$ to this number, in order to stay clear from the noisy region in the PSD. Linear interpolation was then used to find a more precise figure for when the power first dropped below this threshold.

\section{RESULTS}

The resulting Doppler spread as a function of UWB frequency is shown in Fig. 2 for three different time periods. Each line represents the median of all observed Doppler spreads in the time interval indicated in the figure.

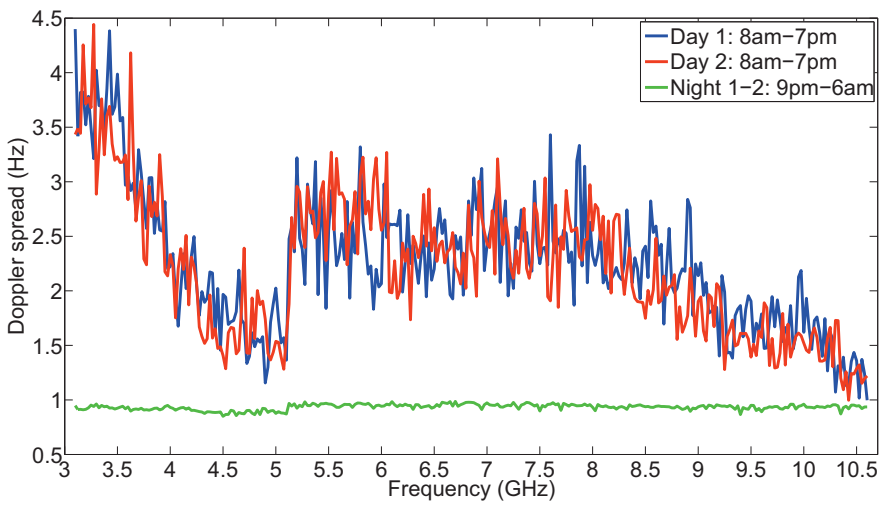

Fig. 2: Doppler spread $(\mathrm{Hz})$ as a function of UWB frequency $(\mathrm{GHz})$

From Fig. 2 we can clearly state that both Doppler spreads measured over two different days show the same frequencydependent behavior. Both tend to decrease heavily from 3.1 to $5.1 \mathrm{GHz}$, where a sudden increase of $1 \mathrm{~Hz}$ can be noticed. From 5.1 to $8 \mathrm{GHz}$, this figure varies between 2 and $3 \mathrm{~Hz}$. A further slight decrease can be noticed from this point on towards the end of the UWB frequency band.

As indicated in [1], instead of just focusing on the Doppler spread alone, it is often more interesting to investigate the behavior of the RMS Doppler spread. This metric provides more insight into the distribution of power, rather than just the width of the power spectrum. The resulting RMS Doppler spread as a function of UWB frequency is shown in Fig. 3.

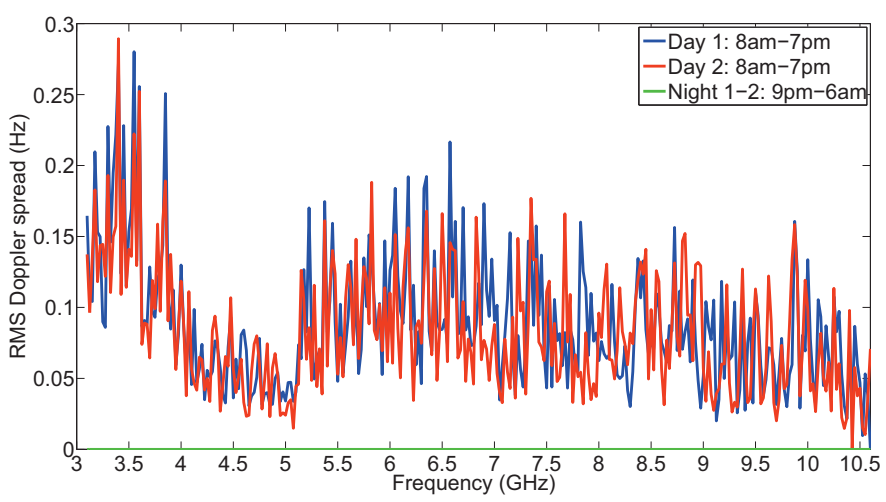

Fig. 3: RMS Doppler spread $(\mathrm{Hz})$ as a function of UWB frequency $(\mathrm{GHz})$

Fig. 3 shows that the RMS Doppler spread is limited to values smaller than $0.3 \mathrm{~Hz}$, indicating that most of the power is concentrated near the center of the PSD. We can again notice a heavy decrease from 3.1 to $5.1 \mathrm{GHz}$, from where a steady behavior can be observed up until $8 \mathrm{GHz}$, followed by a slight decrease towards $10.6 \mathrm{GHz}$.

\section{CONCLUSION}

This work presented an estimate of both the Doppler spread and RMS Doppler spread for UWB indoor communication in an occupied office environment, based on channel sounding measurements ranging from 3.1 to $10.6 \mathrm{GHz}$. A custom made approach was followed in order to estimate both parameters from the channel sounding data. Our measurements indicate a frequency-dependent behavior for both parameters. Consistent values could be measured with respect to time of observation.

Future work could focus on similar measurements performing over a longer time with different antennas in more crowded spaces like railway stations, airports or shopping malls.

\section{ACKNOWLEDGMENT}

Brecht Hanssens is funded by the Institute for the Promotion of Innovation by Science and Technology in Flanders (IWT). This research was supported by the FWO project G027714N and IAP BESTCOM; Belgian network on stochastic modeling, analysis, design and optimization of communication systems.

\section{REFERENCES}

[1] S. J. Howard and K. Pahlavan. Doppler spread measurements of indoor radio channel. Electronics Letters, 26(2):107-109, 1990.

[2] S. Thoen, L. Van der Perre, and M. Engels. Modeling the Channel TimeVariance for Fixed Wireless Communications. Communications Letters, IEEE, 6(8):331-333, 2002.

[3] J. G. Proakis and D. G. Manolakis. Digital Signal Processing (4th Ed.): Principles, Algorithms, And Applications. Pearson Education, 2007. 